\title{
Structural studies on a unique glucosamine kinase unveil a novel enzyme family
}

\author{
J. A. Manso ${ }^{1,2}$, D. Nunes-Costa ${ }^{3,4}$, S. Macedo-Ribeiro ${ }^{1,2}$, N. Empadinhas ${ }^{3,4}$, P. J. B. Pereira ${ }^{1,2}$
}

${ }^{1}$ IBMC-Instituto de Biologia Molecular e Celular, Universidade do Porto, Porto, Portugal, ${ }^{2}$ Instituto de Investigação e Inovação em Saúde, Universidade do Porto, Porto, Portugal, ${ }^{3}$ CNC-Center for Neuroscience and Cell Biology, University of Coimbra, Coimbra, Portugal, ${ }^{4}$ PhD Program in Experimental Biology and Biomedicine (PDBEB), University of Coimbra, Coimbra, Portugal

$$
\text { jose.manso@i3s.up.pt }
$$

The discovery of novel enzymes from antibiotic production pathways is nowadays a topic of utmost importance due to worldwide concerns with the increased resistance of pathogenic bacteria to antibiotics. In this work, we used a combination of X-ray crystallography, SAXS, and biochemical studies to identify the molecular fingerprints for a novel glucosamine kinase (GlcNK) family potentially implicated in antibiotic biosynthesis in Actinobacteria. We determined the high-resolution structure of a bacterial GlcNK in apo form and in complex with its biological substrates, providing unparalleled structural evidence of a transition state of the phosphoryl-transfer mechanism in this unique family of enzymes (PDB IDs 6HWJ, 6HWK and 6HWL; Fig. 1a-c). Conservation of glucosamine-contacting residues across a large number of uncharacterized proteins unveiled a specific glucosamine binding sequence motif. As result, a new UniProt annotation rule was created (MF_02218; Fig. 1d). The structural characterization of this enzyme provides new insights into the role of these unique GlcNKs as the missing link for the incorporation of environmental glucosamine to the metabolism of important intermediates in antibiotic production [1].

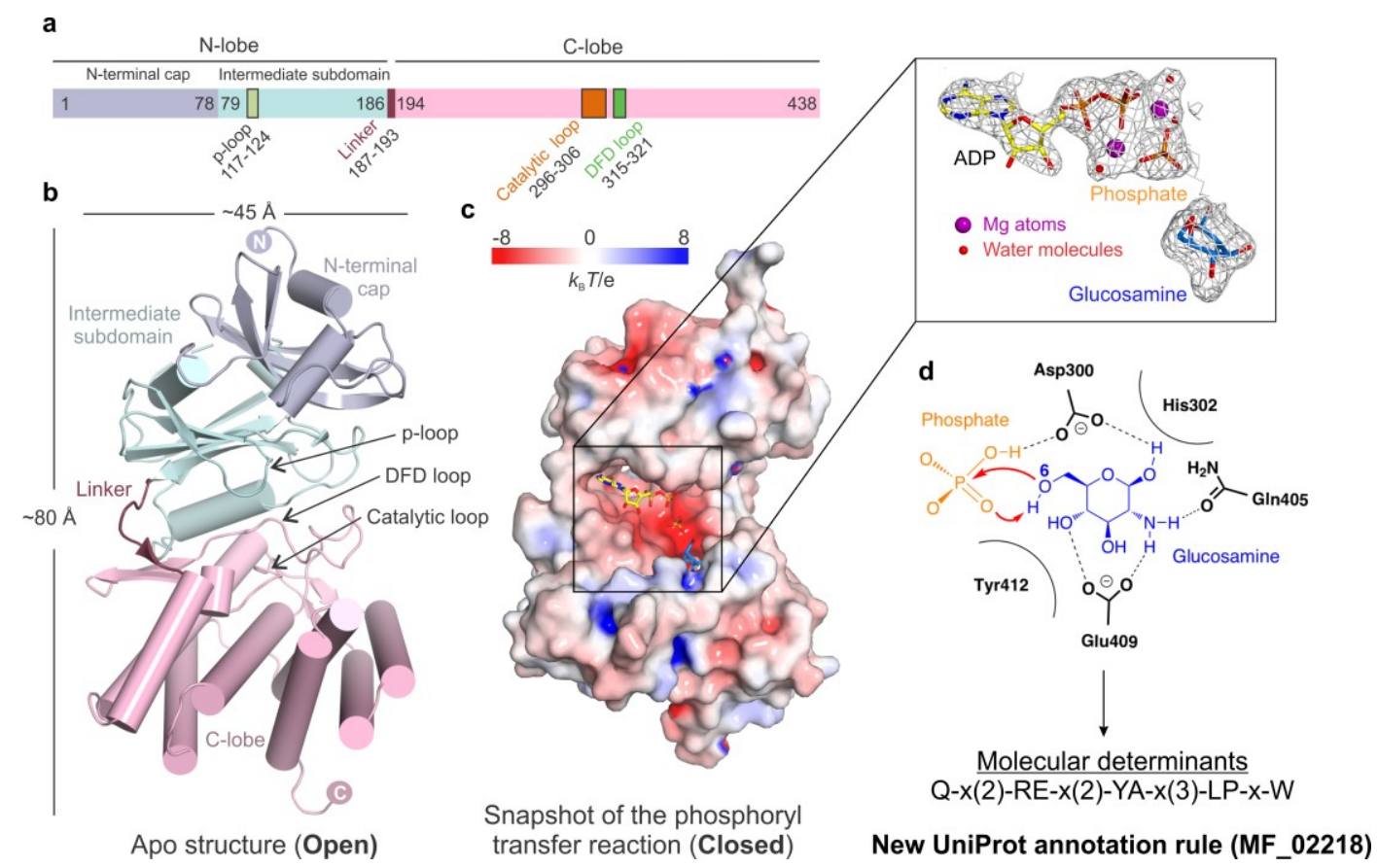

Figure 1. Molecular fingerprints for a novel GlcNK family in Actinobacteria. a) Structural organization of GlcNK. b-c) Cartoon and solid surface representations of the 3D structure of GlcNK in its apo form and in complex with its biological substrates (electron density map $(2 \mathrm{mFo}-D F \mathrm{c}$ contoured at $1.0 \sigma)$ represented as a grey mesh). d) Scheme of the glucosamine-protein interactions.

[1] Manso, J. A., Nunes-Costa, D., Macedo-Ribeiro, S., Empadinhas, N., Pereira, P. J. B. (2019). mBio. 10, e00239-19.

\section{Keywords: Glucosamine kinase; antibiotic production; X-ray crystallography; small-angle X-ray scattering}

We acknowledge ALBA-CELLS and ESRF for access to synchrotron radiation facilities. This work was financed by FEDER - Fundo Europeu de Desenvolvimento Regional funds through the COMPETE 2020 - Operational Programme for Competitiveness and Internationalisation (POCI), Portugal 2020, and by Portuguese funds through FCT - Fundação para a Ciência e a Tecnologia/Ministério da Ciência, Tecnologia e Ensino Superior in the framework of the project "Institute for Research and Innovation in Health Sciences" (POCI-01-0145-FEDER-007274).

Acta Cryst. (2021), A77, C249 